\title{
Conductive link between cryocooler and magnet in cryogen-free LTS magnet system
}

\author{
Yeon Suk Choi* \\ Korea Basic Science Institute, Daejeon, Korea
}

(Received 4 December 2013; revised or reviewed 18 December 2013; accepted 19 December 2013)

\begin{abstract}
The conductive link is used as a cooling medium between a cryocooler and magnet in a cryogen-free superconducting magnet system. The low temperature superconducting (LTS) magnet has one solenoidal configuration with a metal former which has a 52 $\mathrm{mm}$ room temperature bore. The superconducting coil is installed in the cryostat maintaining high vacuum and cooled by a two-stage cryocooler. In order to maintain the operating temperature of magnet at the designed level, the cold head temperature of the cryocooler must be lower so that heat can be removed from the superconducting coil. Also, temperature difference is occurred between the magnet and cryocooler and its magnitude is dependent upon the contact resistance at the interfacial surface between metals in the conductive link. In the paper, the performance of the LTS magnet is investigated with respect to the conductive link between the magnet former and the cold head of the cryocooler. The effects of the contact pressure and interfacial materials on the temperature distribution along the conductive link are also presented.
\end{abstract}

Keywords : Conductive link, contact resistance, cryogen-free, superconducting magnet

\section{INTRODUCTION}

Instead of liquid helium or nitrogen, a cryocooler is used as a heat sink in the cryogen-free superconducting magnet system. Since no cryogenic liquid is used, the cryogen-free superconducting magnets have several advantages, such as compactness, ease handling, free rotation of magnetic field axes, and ease access toward the central magnetic field [1]-[5]. The first practical cryogen-free superconducting magnet has been developed in the early 1990s using a Gifford-McMahon (GM) cryocooler [6]. The superconducting magnet system was consisted of a $\mathrm{Nb} 3 \mathrm{Sn}$ superconducting coil and $0.5 \mathrm{~W}$ of cryocooler, and generated $4.7 \mathrm{~T}$ in a $38 \mathrm{~mm}$ room temperature bore. Since the success, the developments have been carried out to get a higher magnetic field, resulting in the increased size of the superconducting magnet [7].

In a cryogen-free superconducting magnet system as shown in Fig. 1, a cryocooler is employed as a heat sink to cool the magnet down to the designed temperature. Since the cold head of a cryocooler has positional restriction, it needs to be located in a low magnetic field area to avoid degradation of its cooling capacity [8]. A conductive link is required between the superconducting magnet and cryocooler in the cryogen-free superconducting magnet system. The metallic material is generally used as a conductive link in the system. Even though the metallic material with high thermal conductivity, such as oxygen free high conductivity copper, is employed, the temperature

\footnotetext{
* Corresponding author: ychoi@kbsi.re.kr
}

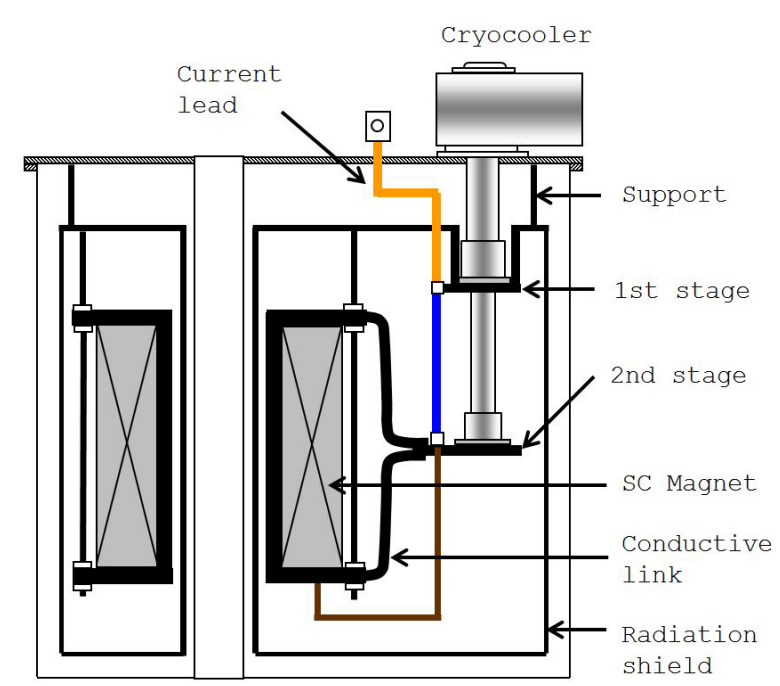

Fig. 1. Representative schematic of a cryogen-free superconducting magnet system.

gradient is inevitable between the magnet and cryocooler mainly due to the thermal contact resistance at the interface between metals. Fig. 2 shows the representative schematic of contact resistance between the superconducting magnet and cryocooler in the cryogen-free superconducting magnet system. As shown in Fig. 2, there are several contact surfaces between metals in the system and it may reduce the thermal stability of magnet because of the small temperature margin.

The low temperature superconducting magnet continuously refrigerated by a cryocooler is presented in 


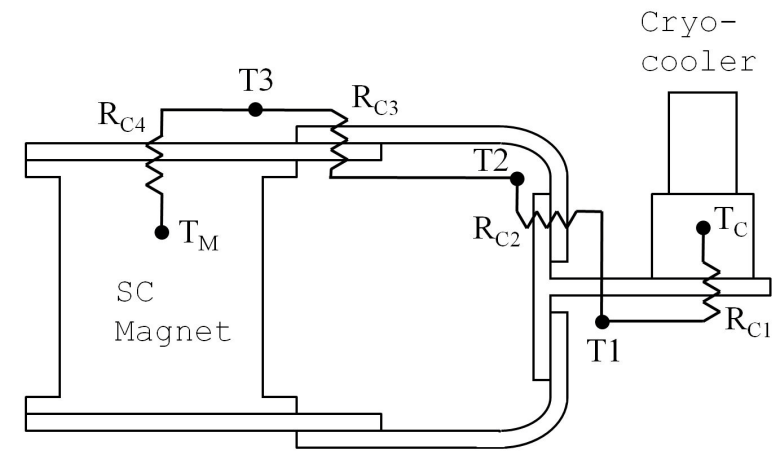

Fig. 2. Schematic of contact resistance between superconducting magnet and cryocooler.

this paper, especially focused on the conductive link. The cryogen-free superconducting magnet and cooling system is fabricated and the initial cool-down process is performed. The temperature variation along the conductive link is quantitatively discussed with respect to the thermal contact resistance between metals measured by precise device. The effect of contact resistance in the conductive link on the cooling performance of the LTS magnet is also presented.

\section{CRYOGEN-FREE SUPERCONDUCTING MAGNET SYSTEM}

Fig. 3 is the 3-dimensional drawing of the cryogen-free superconducting magnet system, showing the L-shaped conductive link. The low temperature superconducting magnet was fabricated using niobium-titanium multi-filamentary core of $0.9 \mathrm{~mm}$ diameter wire by wet winding method. The coil was wound on a metal former which has a $1 \mathrm{~mm}$ slot along the axis to reduce eddy losses during magnet charging process. The total length of niobium-titanium wire was $1700 \mathrm{~m}$ and the overall mass of the magnet was approximately $8.7 \mathrm{~kg}$. The fully assembled magnet has a height of $175 \mathrm{~mm}$ and a diameter of $130 \mathrm{~mm}$. The main parameters of the cryogen-free superconducting magnet system are summarized in Table I.

The current leads made of HTS tapes were connected between the niobium-titanium coil and the copper end terminals. The first stage of a cryocooler was connected to the HTS leads by several bolts and the second stage of a cryocooler was connected to the niobium-titanium magnet coil by soft soldering. The dimension of copper lead is optimized to minimize the thermal load, considering operational current. Table I provides the dimensions of the optimized copper lead. For the HTS section, the commercial HTS lead from HTS-110 [9] is selected for this application. The operating current at $64 \mathrm{~K}$ is $250 \mathrm{~A}$ and conductive heat leak per pair is $76 \mathrm{~mW}$.

The superconducting magnet former is thermally connected to the second stage cold head of the cryocooler through the flexible tinned copper braids. The cooling capacity of a GM cryocooler is $1 \mathrm{~W}$ at $4.2 \mathrm{~K}$. The radiation shield made of aluminium plate is thermally connected to

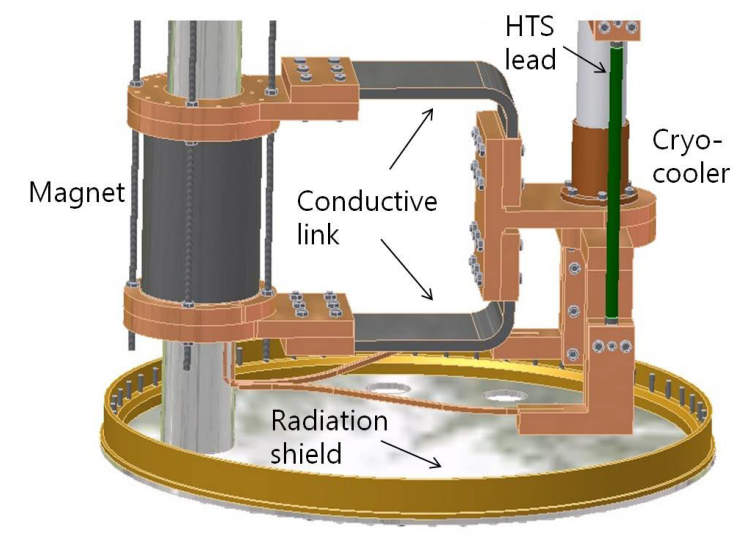

Fig. 3. Drawing of SC magnet system, showing L-shaped conductive link.

TABLE I

PARAMETERS OF A CRYOGEN-FREE SC MAGNET SYSTEM.

\begin{tabular}{|c|c|c|c|c|}
\hline \multicolumn{3}{|l|}{ Parameter } & VALUE & UNITS \\
\hline \multirow[t]{6}{*}{ Magnet } & \multicolumn{2}{|c|}{ Central field } & 3 & $\mathrm{~T}$ \\
\hline & \multicolumn{2}{|l|}{ Conductor } & $\mathrm{NbTi}$ & - \\
\hline & \multicolumn{2}{|c|}{ Outer diameter } & 130 & $\mathrm{~mm}$ \\
\hline & \multicolumn{2}{|l|}{ Height } & 175 & $\mathrm{~mm}$ \\
\hline & \multicolumn{2}{|l|}{ Coil turn } & $184 \times 28$ & - \\
\hline & \multicolumn{2}{|c|}{ Operating current } & 165 & $\mathrm{~A}$ \\
\hline \multirow[t]{6}{*}{ Current lead } & \multirow[t]{3}{*}{ Copper } & Eff. area. & 100 & $\mathrm{~mm}^{2}$ \\
\hline & & Eff. length & 1300 & $\mathrm{~mm}$ \\
\hline & & Heat load & 12.5 & $\mathrm{~W} /$ pole \\
\hline & \multirow{3}{*}{$\begin{array}{l}\text { HTS } \\
\text { (BSCCO) }\end{array}$} & Dia. (body) & 11.13 & $\mathrm{~mm}$ \\
\hline & & Length & 305 & $\mathrm{~mm}$ \\
\hline & & Heat load & 76 & $\mathrm{~mW}$ \\
\hline \multirow[t]{3}{*}{ Cryostat } & \multicolumn{2}{|l|}{ Diameter } & 600 & $\mathrm{~mm}$ \\
\hline & \multicolumn{2}{|l|}{ Height } & 800 & $\mathrm{~mm}$ \\
\hline & \multicolumn{2}{|c|}{ Warm bore dia. } & 52 & $\mathrm{~mm}$ \\
\hline
\end{tabular}

the first stage cold head by several bolts. The heat loads are $750 \mathrm{~mW}$ in total, including support conduction, thermal radiation, current lead, and infiltration heat due to instrumentation. The 2nd stage temperature of the cryocooler is $3.2 \mathrm{~K}$ from the cooling capacity of a two-stage GM cryocooler [10], using the total heat loads.

The superconducting magnet is enclosed in a vacuum vessel and conductively cooled by a GM cryocooler. Temperatures in the magnet system are measured with resistance temperature detector. All temperatures in the system are recorded at interval of 10 second with an automated data acquisition system operated through the GPIB communication module of LabView ${ }^{\mathrm{TM}}$ software.

The initial cooling of superconducting magnet is then carried out using a cryocooler. Fig. 4 shows the temperature history of first cold head, radiation shield, second cold head and superconducting magnet after turning on a cryocooler. The temperature of superconducting magnet is stabilized after 16 hours running of cryocooler. The temperature of second cold head was $3.2 \mathrm{~K}$ and that of superconducting magnet was $6.1 \mathrm{~K}$. For practical contact between metals the interface is irregular enough that only intermittent contact could occur. The actual contact area 


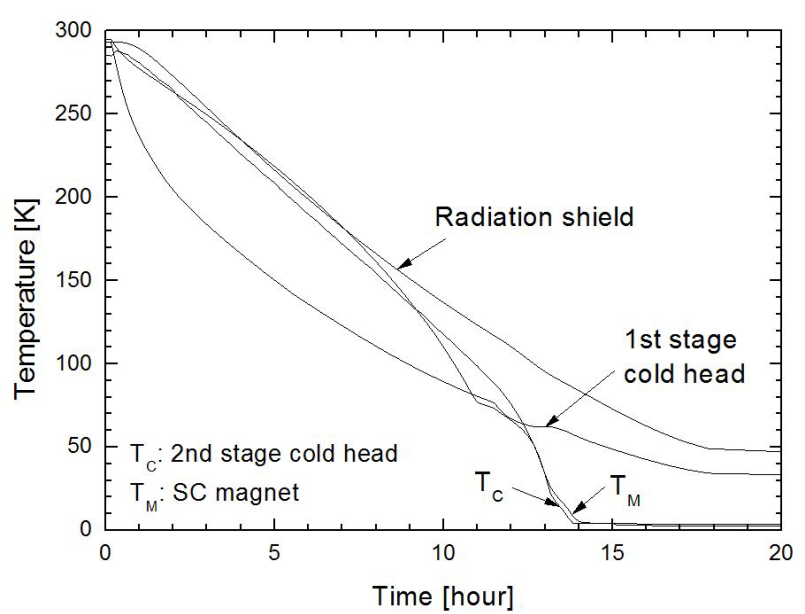

Fig. 4. Initial cooldown process of cryogen-free superconducting magnet system.

could be increased by the interfacial films or bonding agents, resulting in the increasing of the thermal conductance [11].

\section{PERFORMANCE TEST}

In the cryogen-free superconducting magnet system, the copper is most commonly used as a conductive link. The thermal contact resistance between copper plates, therefore, is measured to investigate the quantitative value. Fig. 5 presents the thermal contact resistance with respect to the temperature as a function of applied contact pressure. The surface roughness of copper plate was measured and its average value was $697 \mathrm{~nm}$. The details of the experiment measuring the thermal contact resistance is described in [12]. The thermal contact resistance increases as the operating temperature decreases. When the contact pressures are 7,14 and $21 \mathrm{MPa}$, the values of thermal contact resistance are $2.74 \times 10^{-4}, 1.38 \times 10^{-4}$ and $8.73 \times 10^{-5}$ $\mathrm{m}^{2} \mathrm{~K} / \mathrm{W}$ at $4 \mathrm{~K}$, respectively. The contact resistance decreases as the contact pressure increases. The thermal conductance of any interface is a function of the actual contacting area and the physical properties of the mating materials [13]. Therefore, high contact pressure can increase the actual contacting area of the interface. Compared to the reference data, the decrease rate of thermal contact resistance is getting smaller as contact pressure increases.

The insertion of the interfacial material between metal surfaces can reduce the thermal contact resistance. Several runs were performed with different interfacial materials under presumably identical conditions. Fig. 6 shows the thermal contact resistance variation when the interfacial material was inserted between copper plates when the contact pressure was $7 \mathrm{MPa}$. The indium sheet, insulation sheet and thermal grease were used as interfacial materials to investigate the effect on the thermal contact resistance. When the indium sheet and thermal grease were used the thermal contact resistance decreased. However, the thermal

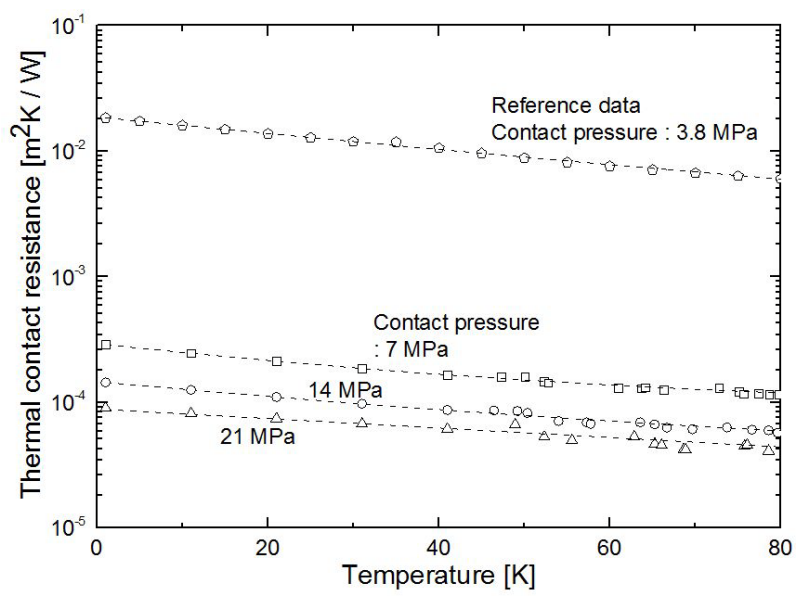

Fig. 5. Thermal contact resistance with respect to the temperature as a function of contact pressure.

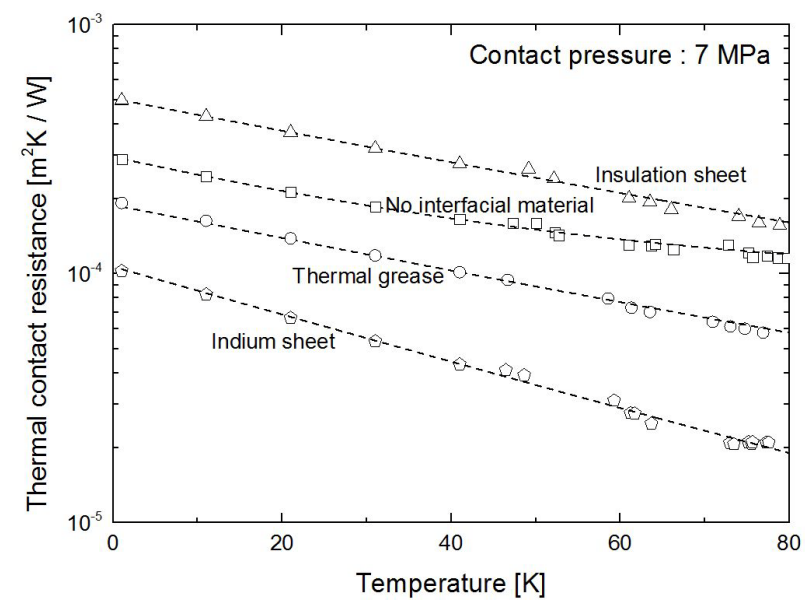

Fig. 6. Variation of thermal contact resistance as function of interfacial materials (contact pressure: $7 \mathrm{MPa}$ ).

contact resistance increased when the electrical insulation sheet was used. The insulation sheet is generally used where the electrical insulation is required in the cryogen-free superconducting magnet system.

The surface of metal plate is not perfectly flat, so some vacancies can exist. The interfacial material which has a high thermal conductivity could enlarge the actual contact area and increase the contact pressure at the same time by filling the vacancies. The thermal contact resistances at $4 \mathrm{~K}$ were $4.76 \times 10^{-4}, 2.74 \times 10^{-4}, 1.83 \times 10^{-4}$ and $9.62 \times 10^{-5}$ $\mathrm{m}^{2} \mathrm{~K} / \mathrm{W}$ when the interfacial materials were insulation sheet, no material, thermal grease and indium sheet, respectively. No material means that it is pure metal-to-metal contact. They were decreased as temperature increased. In the cryogen-free superconducting magnet system, the insulation sheet composed of non-metal is used as a medium for electrical insulation but large temperature gradient is inevitable across the insulation sheet.

Based upon the quantitative value of thermal contact resistance between metals, the temperature distribution between the magnet and cryocooler is estimated. Fig. 7 shows the temperatures along the conductive link in the 


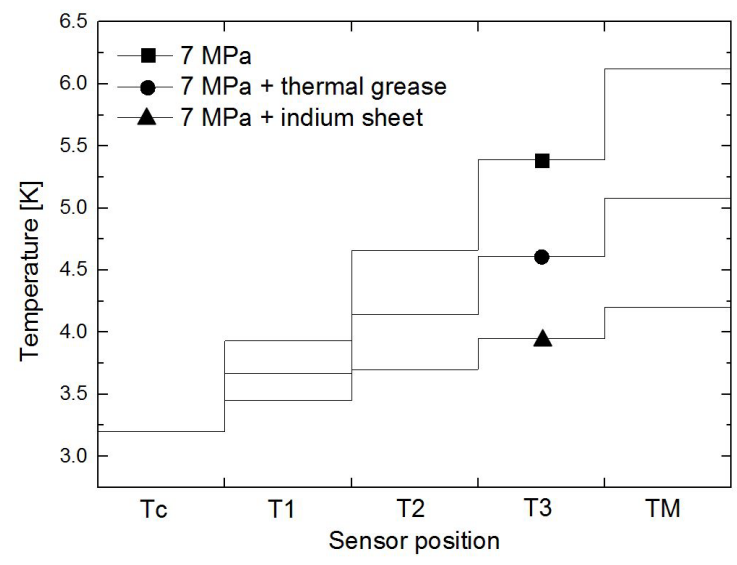

Fig. 7. Estimated temperatures along the conductive link in the cryogen-free superconducting magnet system.

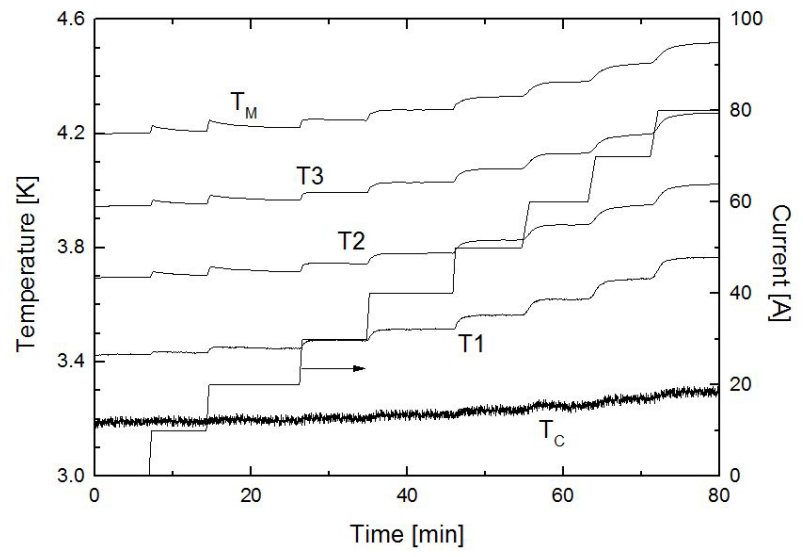

Fig. 8. Temperatures and supplied current with respect to the elapsed time during magnet charging process.

cryogen-free superconducting magnet system. We assumed that the temperature difference was occurred at the contact surface and the measured contact resistance was used for temperature gradient estimation. The cold head temperature is $3.2 \mathrm{~K}$ and that of magnet is $6.12 \mathrm{~K}$, which agrees well with our measurements, described in previous section. The estimated value of magnet is $4.2 \mathrm{~K}$ when the indium sheet is inserted between metals and $7 \mathrm{MPa}$ of contact pressure is applied.

The temperatures along the conductive link and supplied current during magnet excitation are shown in Fig. 8. The indium sheet was inserted at the contact surface and the current ramping rate was set to be $30 \mathrm{~A} / \mathrm{min}$. The temperature of the superconducting magnet was $4.2 \mathrm{~K}$ and increased with the charging current. The magnet was trained by repeating the charging and discharging process several times. The temperature of the magnet was $4.9 \mathrm{~K}$ when the $165 \mathrm{~A}$ of current was supplied, and the designed magnetic field in the center of the room temperature bore was generated. The decreasing of the temperature gradient between superconducting magnet and cryocooler is acquired by reduced thermal contact resistance resulting from extended contact area and interfacial indium sheet.

\section{CONCLUSIONS}

In the cryogen-free low temperature superconducting magnet system, the temperature gradient between the superconducting coils and the cryocooler was observed mainly because of the thermal contact resistance. It was reduced by increasing the interfacial thermal conductance hiring a bonding agent and using a high contact pressure. An indium sheet between metals was an effective interfacial material and temperature distribution was estimated using a quantitative value of contact resistance. For the temperature distribution in the superconducting magnet system, good agreement was observed between measurement and calculation. The measured values of thermal contact resistance should contribute to design the cryogenic device cooled by a cryocooler.

\section{ACKNOWLEDGMENT}

The work was supported by the KBSI Grant T33424.

\section{REFERENCES}

[1] H. Morita, M. Okada, K. Tanaka, J. Sato, H. Kitaguchi, H Kumakura, K. Togano, K. Itoh, and H. Wada, "10 T conduction cooled Bi-2212/Ag HTS solenoid magnet system," IEEE Trans. App. Supercond., vol. 11, pp. 2523-2526, 2001.

[2] K. Watanabe, and K. Takahashi, "Cryogen-free hybrid magnet for magnetic levitation," Physica C, vol. 386, pp. 485-489, 2003.

[3] Q. Wang, L. Yan, B. Zhao, S. Song, and Y. Lei, "Development of wide-bore conduction-cooled superconducting magnet system for material processing applications," IEEE Trans. App. Supercond., vol. 14, pp. 372-375, 2004.

[4] Y. Dai, L. Yan, B. Zhao, S. Song, Y. Lei, and Q. Wang, "Tests on a 6 T conduction-cooled superconducting magnet," IEEE Trans. App. Supercond., vol. 16, pp. 961-964, 2006.

[5] S. Katano, N. Minakawa, T. Hasebe, and J. Sakuraba, "New cryocooler-cooled superconducting magnet: A $13.5 \mathrm{~T}$ high-field split-pair coil magnet for neutron scattering," Physica B, vol. 385-386, pp. 1300-1302, 2006.

[6] K. Watanabe, Y. Yamada, J. Sakuraba, F. Hata, C.K. Chong, and T. Hasebe, "(Nb,Ti) $)_{3} \mathrm{Sn}$ superconducting magnet operated $11 \mathrm{~K}$ in vacuum using high-Tc current lead," Jpn. J. Appl. Phys., vol. 32, pp. 488-490, 1993.

[7] G. Nishijima, S. Awaji, S. Hanai, and K. Watanabe, "18.1 T cryocooled superconducting magnet with a Bi223 high-Tc insert," Fusion Engineering and Design, vol. 81, pp. 2425-2432, 2006.

[8] Y.S. Choi, D.L. Kim, T.A. Painter, W.D. Markiewicz, B.S. Lee, H.S Yang, and J.S. Yoo, "Closed-loop cryogenic cooling for a $21 \mathrm{~T}$ FT-ICR magnet system,” IEEE Trans. App. Supercond., vol. 18, pp. 1471-1474, 2008

[9] HTS-110 Co., "HTS-110 product documentation," Westborough, MA [Online]. Available: http://www.hts-110.com.

[10] Sumitomo Heavy Ind., "Sumitomo product documentation," Allentown, PA [Online]. Available: http:// www.shicryogenics.com

[11] S.W. Van Sciver, M.J. NillesE, and J. Pfotenhaur, "Thermal and electrical contact conductance between metals at low temperature," in Space Cryogenic Workshop, Berlin, pp. 37-48, 1984.

[12] M.S. Kim and Y.S. Choi, "Measurement of thermal contact resistance at $\mathrm{Cu}-\mathrm{Cu}$ interface," Progress in Superconductivity and Cryogenics, vol. 15, no. 2, pp. 48-52, 2013.

[13] J. Yu, A.L. Yee, and R.E. Scheall, "Thermal conductance of $\mathrm{Cu} / \mathrm{Cu}$ and $\mathrm{Cu} / \mathrm{Si}$ interface from $85 \mathrm{~K}$ to $300 \mathrm{~K}$," Cryogenics, vol. 32, pp. 610-615, 1992. 